\title{
OPEN A novel algorithm to detect non-wear time from raw accelerometer data using deep convolutional neural networks
}

\begin{abstract}
Shaheen Syed ${ }^{1 凶}$, Bente Morseth ${ }^{2}$, Laila A. Hopstock ${ }^{3}$ \& Alexander Horsch ${ }^{1}$
To date, non-wear detection algorithms commonly employ a 30,60, or even 90 mins interval or window in which acceleration values need to be below a threshold value. A major drawback of such intervals is that they need to be long enough to prevent false positives (type l errors), while short enough to prevent false negatives (type II errors), which limits detecting both short and longer episodes of non-wear time. In this paper, we propose a novel non-wear detection algorithm that eliminates the need for an interval. Rather than inspecting acceleration within intervals, we explore acceleration right before and right after an episode of non-wear time. We trained a deep convolutional neural network that was able to infer non-wear time by detecting when the accelerometer was removed and when it was placed back on again. We evaluate our algorithm against several baseline and existing non-wear algorithms, and our algorithm achieves a perfect precision, a recall of 0.9962 , and an F1 score of 0.9981 , outperforming all evaluated algorithms. Although our algorithm was developed using patterns learned from a hip-worn accelerometer, we propose algorithmic steps that can easily be applied to a wrist-worn accelerometer and a retrained classification model.
\end{abstract}

Accelerometer-based motion sensors have become a popular tool to measure and characterise daily physical activity (PA $)^{1-4}$. The use of accelerometers in research and consumer applications has grown exponentially ${ }^{5}$, as accelerometers offer versatility, minimal participation burden, and relative cost efficiency ${ }^{6-8}$. As a result, accelerometers have become the standard tool for measuring PA in large epidemiological cohort studies ${ }^{9}$.

One essential step in the processing of accelerometer data is the detection of the time the accelerometer is not worn (non-wear time) ${ }^{10}$. Non-wear time can occur during sleep, sport, showering, water-based activities, or simply when forgetting to wear the accelerometer. Non-wear detection algorithms developed for count-based accelerometer data typically look for periods of zero acceleration within specified time intervals, such as 30,60 , or 90 mins intervals ${ }^{11-13}$. Unfortunately, the accuracy of current count-based non-wear algorithms is sub-optimal as they frequently misclassify true wear time as non-wear time (type I error) ${ }^{14}$, especially during episodes of sleep and sedentary behaviour ${ }^{15-18}$.

During recent years, with technological advances, accelerometers are able to record and store raw acceleration data (in gravity units $[g]$ ) over three axes with sample frequencies up to $100 \mathrm{~Hz}$ or more ${ }^{5}$. The use of raw data opens up new analytical methods and, in contrast to count-based methods ${ }^{8}$, could enable a direct comparison of the data obtained from different accelerometer devices ${ }^{5}$. However, the development of non-wear algorithms for raw acceleration data has received little attention, despite the widespread adoption of raw accelerometer sensors in PA related studies. These algorithms typically examine the standard deviation (SD) and acceleration value ranges of the acceleration axes within a certain time interval and associate low values with non-wear time ${ }^{19,20}$. In addition, a recent study has evaluated and proposed other means of determining non-wear time, such as inspecting acceleration values when filtering the data (high-pass filter), or by inspecting changes in tilt angles (slope) ${ }^{21}$.

However, all current algorithms employ a rather long minimum time interval (e.g. 30, 60, or even 90 mins) in which a specific measure (e.g. the SD, vector magnitude unit (VMU) or tilt) needs to be below a threshold value. The underlying rationale for using such a time interval is arguably based on analytic approaches adopted from traditional count-based algorithms $\mathrm{s}^{5}$. A major drawback of algorithms employing a time interval is that

${ }^{1}$ Department of Computer Science, UiT The Arctic University of Norway, Tromsø, Norway. ${ }^{2}$ School of Sport Sciences, Faculty of Health Sciences, UiT The Arctic University of Norway, Tromsø, Norway. ${ }^{3}$ Department of Community Medicine, Faculty of Health Sciences, UiT The Arctic University of Norway, Tromsø, Norway. ${ }^{\square}$ email: shaheen.syed@uit.no 
any non-wear episode shorter than the interval cannot be detected. This would negatively impact the recall (also referred to as sensitivity) performance and can cause an increase in false negatives or type II errors; true non-wear time inferred as wear time. In other words, it is rather safe to assume that an interval of 60 mins of no activity can be considered non-wear time, albeit that this assumption comes at a cost.

To remedy the above, and to fully unlock the potential of raw accelerometer data, this paper explores an analytical method frequently employed in activity type recognition studies. That is, the use of deep neural networks to detect activity types such as jogging, walking, cycling, sitting, and standing ${ }^{22-27}$, as well as more complex activities such as smoking, eating, and falling ${ }^{28,29}$. Following this line of research, we hypothesise that episodes of non-wear time precede and follow specific activities or movements that can be characterised as taking off the accelerometer and placing it back on, and that such activities can be detected through the use of deep neural networks. In doing so, we can distinguish episodes of true non-wear time from episodes that only show characteristics of non-wear time, but are in fact wear time.

We utilised a gold-standard dataset with known episodes of wear and non-wear time constructed from two accelerometers and electrocardiogram (ECG) recordings ${ }^{14}$. This gold-standard dataset contains thus ground-truth labels of wear and non-wear time that were detected by calculating discrepancies between two accelerometers worn at the same time, including one which additionally recorded ECG and derived heart rate ${ }^{14}$. We trained several convolutional neural networks to classify activities that precede and follow wear and non-wear time. In doing so, we aimed to develop a novel algorithm to detect non-wear time from raw acceleration data that can detect non-wear time episodes of any duration, thus removing the need for currently employed time intervals. To evaluate the performance of our algorithm, we compared it with several baseline and previously developed non-wear algorithms that work on raw data ${ }^{19-21}$.

\section{Methods}

Gold-standard dataset. The gold-standard dataset was constructed from a dataset containing raw accelerometer data from 583 participants of the Tromsø Study, a population-based cohort study in the municipality of Tromsø in Norway, and includes seven data collection waves taking place between 1974 and $2016^{30,31}$. Our dataset was acquired in the seventh wave of the Tromsø Study. Troms $\varnothing$ was approved by the Regional Committee for Medical Research Ethics (REC North ref. 2014/940) and the Norwegian Data Protection Authority, and all participants gave written informed consent. The usage of data in this study has been approved by the Data Publication Committee of the Tromsø Study. Furthermore, all methods were carried out in accordance with relevant guidelines and regulations (i.e. Declaration of Helsinki).

The dataset contains, for each of the 583 participants, raw acceleration data recorded by an ActiGraph model wGT3X-BT accelerometer (ActiGraph, Pensacola, FL) with a dynamic range of $\pm 8 g\left(1 g=9.81 \mathrm{~ms}^{-2}\right)$. The ActiGraph recorded acceleration in gravity units $g$ along three axes (vertical, mediolateral and anteroposterior) with a sampling frequency of $100 \mathrm{~Hz}$. In addition, this dataset contains data from the simultaneously worn Actiwave Cardio accelerometer (CamNtech Ltd, Cambridge, UK) with a dynamic range of $\pm 8 g$ that recorded raw acceleration data along three axes, as well as a full single-channel ECG waveform. The dataset consisted of data from 267 (45.8\%) males and $316(54.2 \%)$ females aged 40-84 (mean =62.74; SD = 10.25). The participants had a mean height of $169.81 \mathrm{~cm}(\mathrm{SD}=9.35)$, a mean weight of $78.31 \mathrm{~kg}(\mathrm{SD}=15.27)$ and a mean body mass index of $27.06 \mathrm{~kg} / \mathrm{m}^{2}(\mathrm{SD}=4.25)$.

Based on this dataset, a gold-standard dataset with labelled episodes of true non-wear time was constructed by training a machine learning classifier that focused on discrepancies between the various signals. The procedure is explained in detail in our previous study ${ }^{14}$, and also details information regarding the frequency of non-wear episodes, their duration and distribution over the course of a day. The constructed gold-standard dataset contains start and stop timestamps for episodes of true non-wear time derived from raw triaxial $100 \mathrm{~Hz}$ ActiGraph acceleration data, and will serve as ground truth labels in subsequent steps of our proposed algorithm. Henceforth, the Actiwave Cardio data has not been used since it was only used in the construction of the gold-standard dataset.

Finding candidate non-wear episodes. The proposed raw non-wear detection algorithm works on the basis of candidate non-wear episodes that are defined as episodes of no activity that show characteristics of true non-wear time but cannot yet be classified as true non-wear time. Candidate episodes occur during actual nonwear time, in which a candidate episode becomes an episode of true non-wear time, but they can also occur during sedentary behaviour or sleeping since the accelerometer records no movement for a certain amount of time. For illustrative purposes, acceleration data with several candidate non-wear episodes are shown in the Supplementary Fig. S1.

Candidate non-wear episodes were detected by calculating the SD of the raw triaxial data for each 1-min interval. By visual inspection of the data, a SD threshold of $\leq 4.0 \mathrm{mg}(0.004 \mathrm{~g})$, recognisable by horizontal or flat plot lines, was found appropriate to obtain candidate non-wear episodes. More concretely, lowering this threshold would not detect any episode of physical inactivity, meaning that $4.0 \mathrm{mg}$ is very close to the accelerometer's noise level. Consecutive 1-min intervals were grouped into candidate non-wear episodes. Additionally, a forward and backward pass over the acceleration data for each of the candidate non-wear episode were performed to detect the edges on a 1-s resolution, that is, the exact point in which an episode of no activity (i.e. SD $\leq 4.0 \mathrm{mg}$ ) follows or precedes some activity (i.e. $\mathrm{SD}>4.0 \mathrm{mg}$ ). For each candidate episode, the exact start and stop timestamp on a 1-s resolution was recorded.

Creating features. The next step in the construction of the non-wear algorithm was to detect the activity associated with taking off the accelerometer and putting the accelerometer back on, from background activity (i.e. activity that occurs before and after a candidate non-wear episode that is not true non-wear time). In doing so, 

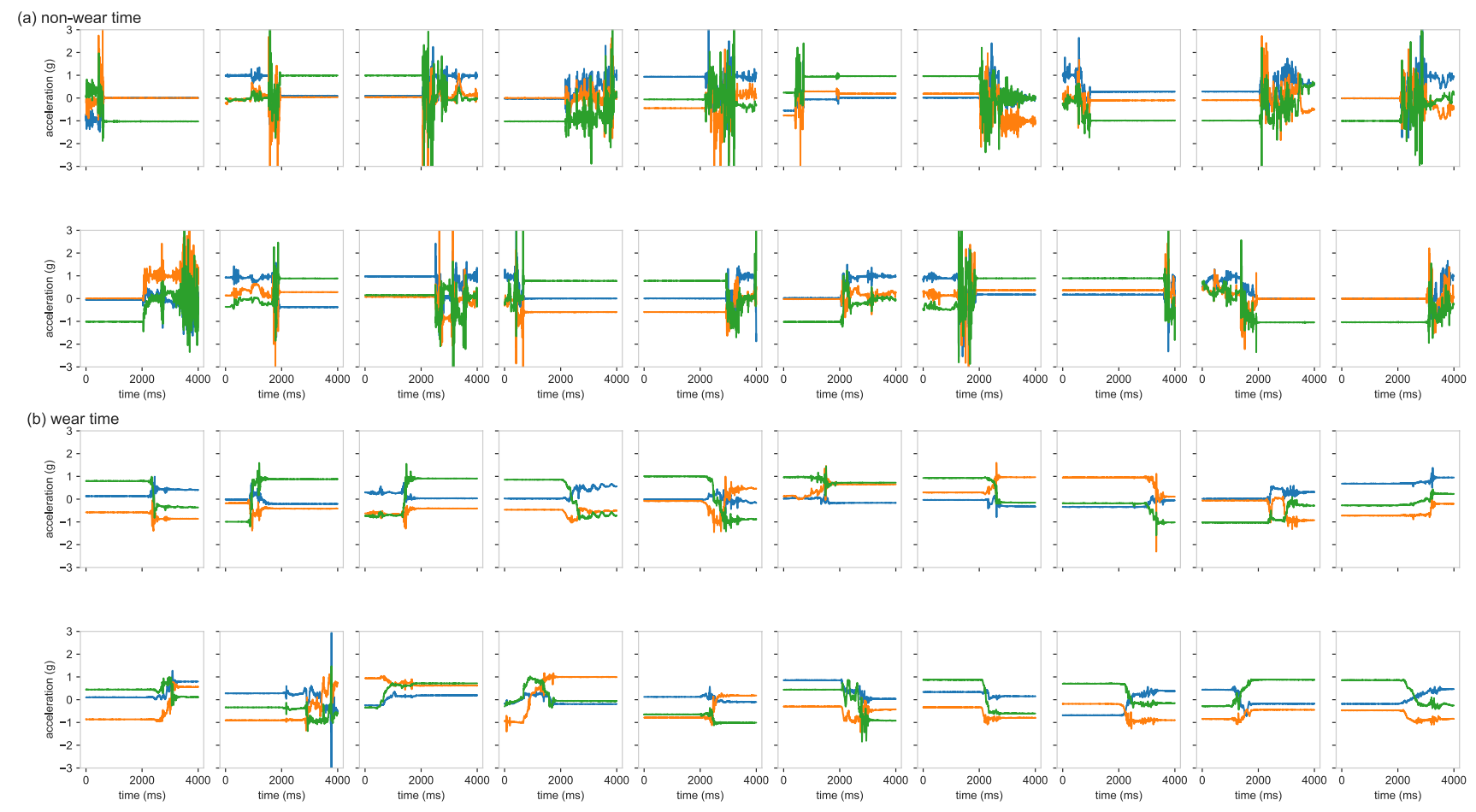

Figure 1. Start or the stop segments of candidate non-wear episodes where features of a length of 2-10 s were extracted; (a) start or stop episodes of true non-wear time, (b) start or stop episodes of wear time.

we extracted a segment or window of raw triaxial acceleration data right before (i.e. preceding) and right after (i.e. following) a candidate non-wear episode, and used the raw triaxial acceleration data as features.

Different features were created by varying the window size from $2-10 \mathrm{~s}$, since the optimal window size was unknown at that point. Technically, a preceding feature is extracted from $t_{\text {start }}-w$ up to $t_{\text {start }}$, where $t_{\text {start }}$ is the start timestamp of the episode in seconds and $w$ is the window size in seconds. The following feature is extracted from $t_{\text {stop }}$ until $t_{\text {stop }}+w$, with $t_{\text {stop }}$ marking the end of an episode; for example, a preceding feature with a 5-s window would yield a $(100 \mathrm{~Hz} \times 5 \mathrm{~s})$ by $(3 \mathrm{axes})=(500 \times 3)$ matrix. In addition, by utilising our labelled goldstandard dataset, each constructed feature was either given the label 0 , if it preceded or followed wear time, or 1 , if it preceded or followed non-wear time; no differences were made between start and stop events. To illustrate this, Fig. 1 displays several start and stop segments from candidate non-wear episodes from where the features were extracted. Importantly, no additional filtering or pre-processing was performed on the raw data, and features belonging to the minority classes were up-sampled by random duplication so as to create a class balanced dataset.

Training a deep neural network. Convolutional neural networks $(\mathrm{CNN})$ are designed to process data in the form of multiple arrays ${ }^{32}$. CNNs are able to extract the local dependency (i.e. nearby signals that are likely to be correlated) and scale invariant (i.e. scale-invariant for different paces or frequencies) characteristics from the feature data ${ }^{27}$. The 1-dimensional (1D) CNN is particularly suitable for signal or sequence data such as accelerometer data ${ }^{32}$ and, to date, $1 \mathrm{D}$ CNNs have successfully been applied for human activity recognition ${ }^{28,33}$, and outperform classical machine learning models on a number of benchmark datasets with increased discriminative power ${ }^{34}$.

A total of four 1D CNN architectures were constructed and trained for the binary classification of our features as either belonging to true non-wear time or to wear time episodes. Figure 2 shows the four proposed architectures labelled V1, V2, V3, and V4. The input feature is a vector of $w \times 3$ (i.e. three orthogonal axes), where $w$ is the window size ranging from 2-10 s (note that a single second contains 100 datapoints for our $100 \mathrm{~Hz}$ data). In total, $9 \times 4=36$ different CNN models were trained. CNN V1 can be considered a basic CNN with only a single convolutional layer followed by a single fully connected layer. CNN V2 and V3 contain additional convolutional layers with different kernel sizes and numbers of filters. Stacking convolutional layers enables the detection of high-level features, unlike single convolutional layers. CNN V4 contains a max pooling layer after each convolutional layer to merge semantically similar features while reducing the data dimensionality ${ }^{32}$. A CNN architecture with max pooling layers has shown varying results, from increased classification performance ${ }^{33}$ to pooling layers interfering with the convolutional layer's ability to learn to down sample the raw sensor data ${ }^{34}$. All proposed CNN architectures have a single neuron in the output layer with a sigmoid activation function for binary classification.

Training was performed on $60 \%$ of the data, with $20 \%$ used for validation and another $20 \%$ used for testing. All models were trained for up to 250 epochs with the Adam optimiser ${ }^{35}$ and a learning rate of 0.001 . Loss was calculated with binary cross entropy and, additionally, early stopping was implemented to monitor the validation loss with a patience of 25 epochs and restore weights of the lowest validation loss. This means that training would terminate if the validation loss did not improve for 25 epochs, and the best model weights would be 


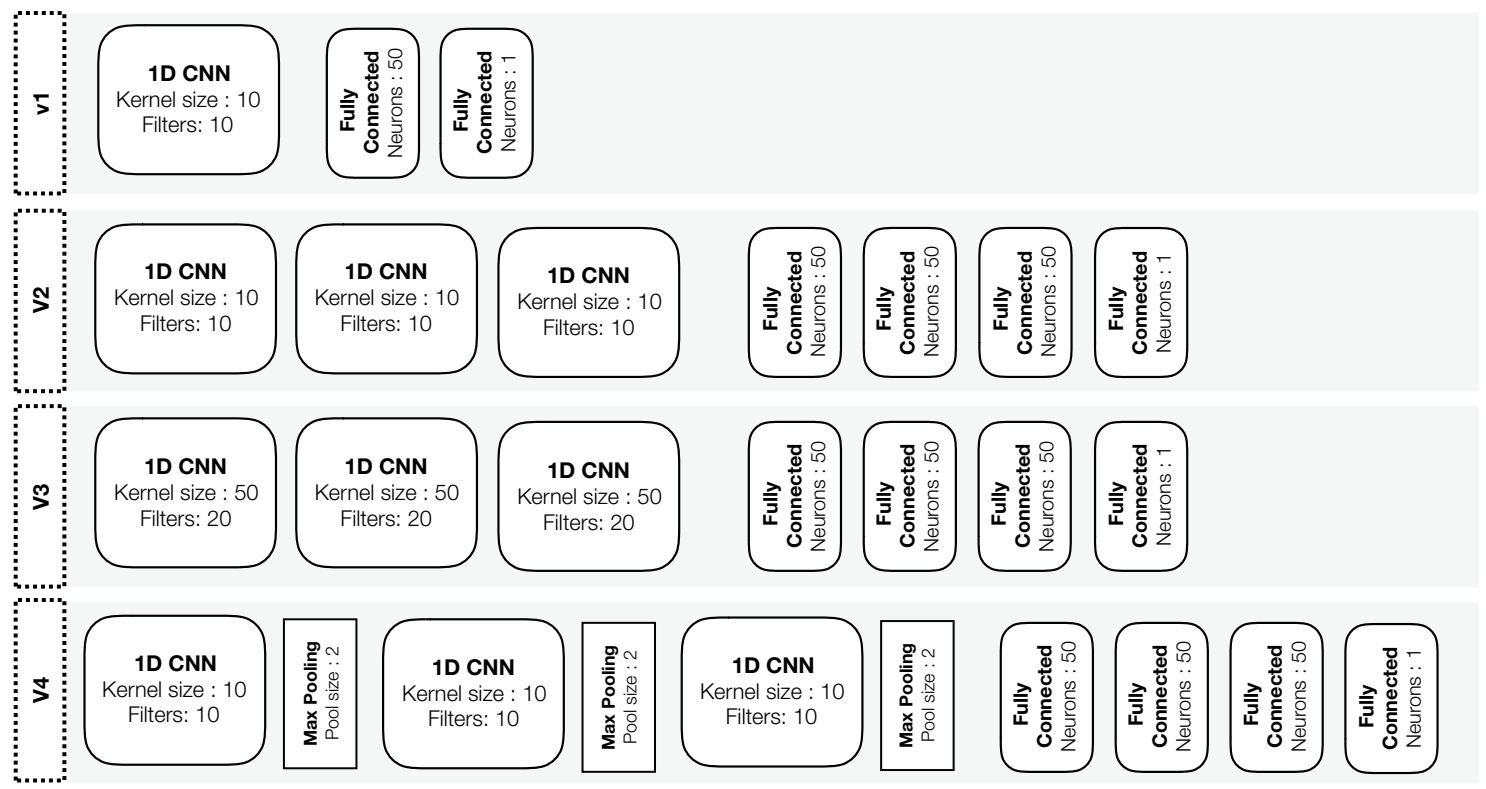

Figure 2. Overview of the four convolutional neural network architectures used for binary classification of start and stop features extracted from wear and non-wear episodes. Before the first fully connected layer, the output data from the previous layer is flattened.

restored. All models were trained on $2 \times$ Nvidia RTX 2080TI graphics cards and programmed in the Python library TensorFlow $(\mathrm{v} 2.0)^{36}$.

Inferring non-wear time from raw acceleration data. At this stage, the trained CNN model can only classify the start and stop windows of a candidate non-wear episode. To fully detect non-wear episodes from raw acceleration data, the following four steps were applied to the algorithm.

Detecting candidate non-wear episodes. As discussed in the previous section, detecting candidate non-wear episodes was based on a forward pass through the raw acceleration data to detect 1-min intervals in which the acceleration has a SD of $\leq 0.004 \mathrm{~g}$. Consecutive 1 -min intervals below this threshold are merged together and considered a single candidate non-wear episode; these episodes formed the basis of the non-wear detection algorithm.

Merging bordering candidate non-wear episodes. Due to artificial movement, a potentially longer non-wear episode might be broken up into several candidate non-wear episodes that are in close proximity to each other. More concretely, the forward search for 1-min intervals with $\leq 0.004 \mathrm{~g}$ SD threshold would not include the 1-min interval in which artificial movement (i.e. a spike in the acceleration) occurred. As a result, when merging together consecutive 1-min intervals, the artificial movement stops this consecutive sequence. The duration of the artificial movement can also vary; for example, moving the accelerometer from the bathroom to the bedroom will take longer than a nudge or touch while the accelerometer lies on a table or nightstand. The first hyperparameter of the algorithm defines the merging length, and five different values of 1,2, 3, 4, and 5 mins were explored; for example, a merging length of 2 mins means that two candidate non-wear episodes that are no more than 2 mins apart are merged together into a single longer candidate non-wear episode.

Detecting the edges of candidate non-wear episodes. Candidate non-wear episodes were detected with a minute resolution (i.e. by using a 1-min interval). However, as it was necessary to determine what happened immediately before (preceding) or immediately after (following) an episode, this resolution was too low. To find the exact timestamp when a candidate non-wear episode started and stopped, a forward and backwards search on a resolution of 1-s was performed. More concretely, the edges were incrementally extended, and the SD was calculated for each extended 1-s interval. When it remained $\leq 0.004 \mathrm{~g}$, the search was continued. This was done forwards to find the exact end of an episode, and backwards to find the exact start of an episode.

It is important to note that the detection of candidate non-wear episodes could have been performed with 1-s intervals, rather than with 1-min intervals. The latter, however, is computationally faster and eliminated the detection of a high number of unwanted candidate non-wear episodes that were only a few seconds in duration.

Classifying the start and stop windows. Activity preceding the start of a candidate non-wear episode was extracted with a window length of 2-10 s, as well as activity that followed from the end of the candidate nonwear episode with a window length of 2-10 s. The exact window length was dependent on the best F1 classification performance measured on the (unseen) test set of the CNN model constructed and described in the 
previous section. After class inference of both the start and stop activity, two logical operators AND and OR were inspected to determine if both sides or a single side resulted in a better detection of true non-wear time. This logical operator was the second hyperparameter to be optimised, and it can take on two different values: AND for both sides and $O R$ for a single side. In addition, candidate non-wear episodes can occur at the start or the end of the acceleration signal and, as such, a preceding or following window cannot be extracted since there is no data. It was also investigated if such cases should default to wear or non-wear time. Default classification for the beginning or end of the activity data was the third hyperparameter to be optimised and this could default to two different values: non-wear time or wear time.

A total of $5 \times 2 \times 2=20$ different combinations of hyperparameter values were tested to explore their classification performance on the gold-standard dataset. To prevent these parameterisations from overfitting to the dataset, their performance on a random sample of $50 \%$ (training set) of the participants from our gold-standard dataset was explored, that is $n=291$, as well as their classification performance on the remaining unseen $50 \%$ (test set) of the participants. In doing so, the aim was to provide hyperparameter values that can generalise to other datasets.

Calculating classification performance. The classification performance is calculated when applying the CNN classification model and the steps described in the previous section to the gold-standard dataset comprising raw acceleration data from 583 participants. True non-wear time inferred as non-wear time contributed to the true positives (TP), and true wear time inferred as wear time contributed to the true negatives (TN). Both TPs and TNs are necessary to obtain a high accuracy of the non-wear time algorithm, as they are the correctly inferred classifications. True non-wear time inferred as wear time contributed to the false negatives (FN), and true wear time inferred as non-wear time contributed to the false positives (FP). The FPs, TPs, FNs, and TNs were calculated by looking at 1-s intervals of the acceleration data and comparing the inferred classification with the gold-standard labels. This process is graphically displayed in Supplementary Fig. S2.

Both FNs and FPs will result in an overall lower accuracy, which is calculated by $\frac{T P+T N}{T P+T N+F P+F N}$. Besides accuracy, we calculated three other classification performance metrics: (i) Precision was calculated as $\frac{T P}{T P+F P}$, (ii) recall as $\frac{T P}{T P+F N}$, and (iii) $\mathrm{F} 1$ as the harmonic mean of precision and recall, $2 \times \frac{\text { precision } \times \text { recall }}{\text { precision }+ \text { recall }}$. Recall represents the fraction of correctly inferred non-wear time in relation to all the true non-wear time-in medicine, this is also known as sensitivity. Precision shows the fraction of correctly inferred non-wear time in relation to all inferred non-wear time.

Evaluating classification performance. Our proposed non-wear algorithm was evaluated against the performance of several baseline and existing non-wear detection algorithms ${ }^{19,20}$. These baseline algorithms employ a similar analytical approach commonly found in count-based algorithms ${ }^{11-13}$, that is, detecting episodes of no activity by using an interval of varying length.

The first baseline algorithm detected episodes of no activity when the acceleration data of all three axes had a SD threshold of $\leq 0.004 \mathrm{~g}, \leq 0.005 \mathrm{~g}, \leq 0.006 \mathrm{~g}$, and $\leq 0.007 \mathrm{~g}$ and the duration did not exceed an interval length of $15,30,45,60,75,90,105$, or 120 mins. A similar approach was proposed in another recent study as the $S D \_X Y Z$ method $^{21}$, although the authors fixed the threshold to $13 \mathrm{mg}$ and the interval to $30 \mathrm{mins}$ for a wrist worn accelerometer. Throughout this paper, the first baseline algorithm is referred to as the XYZ algorithm.

The second baseline algorithm was similar to the first baseline algorithm, albeit that the SD threshold was applied to the vector magnitude unit (VMU) of the three axes, where VMU is calculated as $\sqrt{a c c_{x}^{2}+a c c_{y}^{2}+a c c_{z}^{2}}$, with $a c c_{x}, a c c_{y}$, and $a c c_{z}$ referring to each of the orthogonal axes. A similar approach has recently been proposed as the $S D_{-} V M U$ algorithm ${ }^{21}$. Throughout this paper, this baseline algorithm is referred to as the VMU algorithm.

Last, our algorithm is evaluated against the Hees algorithms (details of which can be found in the open source library $\mathrm{GGIR}^{37}$ ) with a 30 mins interval ${ }^{19}$, a 60 mins interval ${ }^{20}$, and a version with optimised hyperparameters and a 135 mins interval ${ }^{14}$. Throughout this paper, these three algorithms are referred to as HEES_30,HEES_60, and HEES_135, indicating their interval length in minutes. Additionally, the sliding window used in the Hees algorithms has been lowered to $1 \mathrm{~min}$, instead of the default $15 \mathrm{mins}$, to make it similar to the sliding window used in the other evaluated algorithms.

\section{Results}

Convolutional neural network. Figure 3 presents the classification performance of the four evaluated CNN architectures. The V2 CNN architecture obtains near perfect F1 scores on the training (60\%), validation $(20 \%)$ and test set $(20 \%)$ with window sizes ranging from 3-7 s. Also, with similar training, validation, and test scores, the models show no signs of overfitting to the training data. The V2 architecture also outperforms the V1, V3, and V4 proposed architectures in terms of accuracy, precision, recall, and F1. The simpler V1 architectureconsisting of a single convolutional layer and a single fully connected layer-outperforms the more complex architectures V3 and V4, though all architectures were trained for a sufficient number of epochs. This improvement holds for all datasets (training, the validation, and test set). The V4 architecture, that implements max pooling layers as a way to downsample the features and is otherwise identical to V2, does not seem to perform as well as the V2 architecture in terms of performance measured during the training, validation, and test sets.

Looking at the V2 architecture, a window size of 3 s provides a marginal increase in F1 performance on the test set (0.995) when compared to the $2 \mathrm{~s}$ window (0.987). Further increasing the window to $7 \mathrm{~s}$ shows a very minor increase in F1 performance on the test set (0.996), however, taking into account the $95 \%$ confidence interval for the $7 \mathrm{~s}$ window $( \pm 0.00512)$, the difference between the CNN models with a 3-6 s window is not statistically 

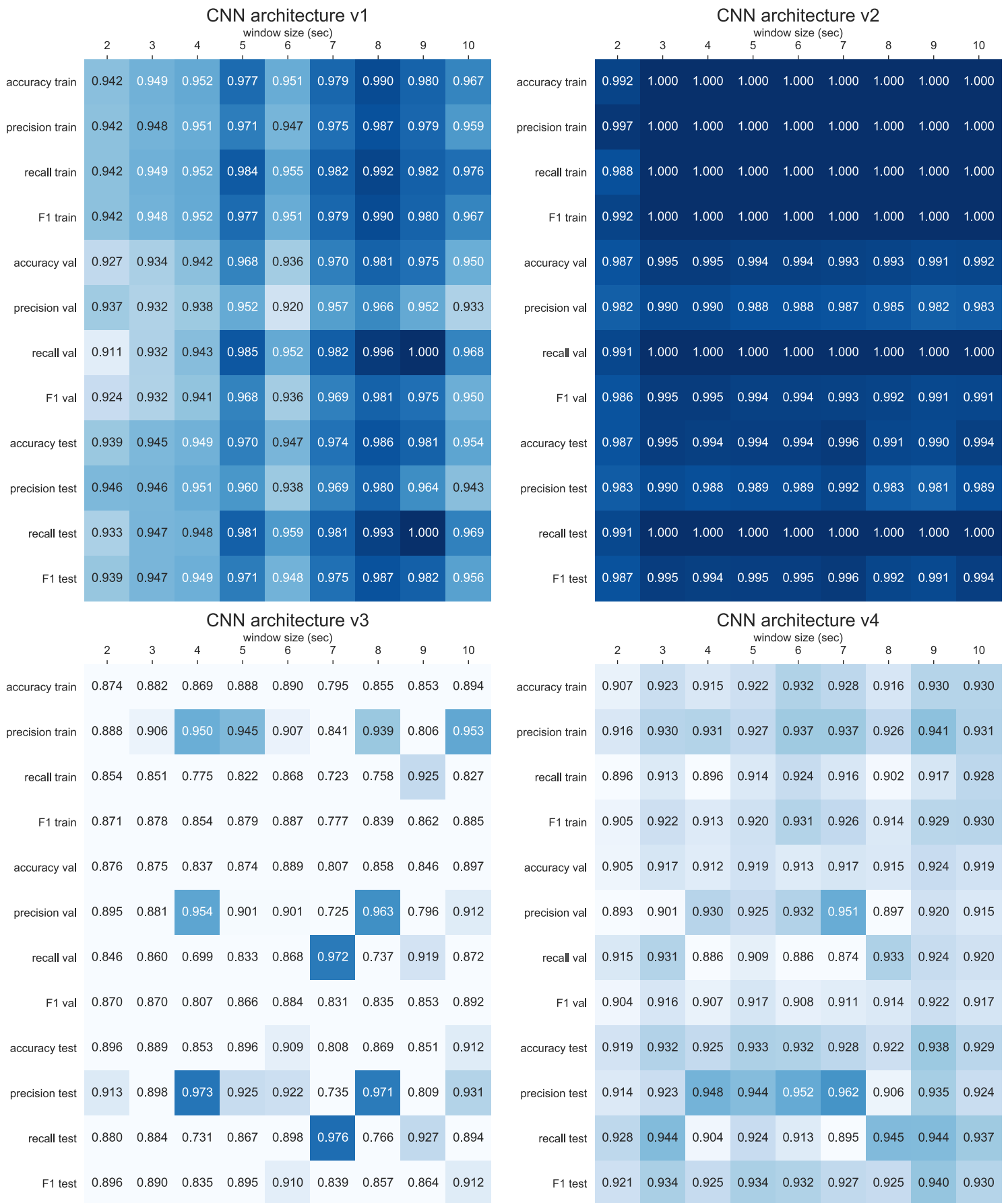

Figure 3. Accuracy, precision, recall, and F1 performance metrics for training data (60\%), validation data $(20 \%)$, and test data $(20 \%)$ for the four architectures evaluated. All CNN models were trained for a total of 250 epochs with early stopping enabled, a patience of 25 epochs, and restoring of the best weights when the validation loss was the lowest.

significant. For the remainder of the results, the V2 CNN model with a window size of $3 \mathrm{~s}$ was selected as the $\mathrm{CNN}$ model to infer if start and stop segments of candidate non-wear time belong to true non-wear time or to wear time episodes. Furthermore, by using a 3-s window, compared to a 7-s window, there is a reduction in the input feature dimensions from $(700 \times 3$ axes $)$ to $(300 \times 3$ axes $)$ for $100 \mathrm{~Hz}$ data, resulting in the CNN model having 144,031 parameters instead of 344,031. An overview of the training and validation loss, including the performance metrics accuracy, precision, recall, F1, and area under the curve (AUC) are presented in the Supplementary Fig. S3.

Non-wear time algorithm hyperparameters. The ability to classify start and stop segments of a candidate non-wear episode is one function of the proposed algorithm. As outlined in the "Methods" section, there 


\begin{tabular}{|c|c|c|c|c|c|c|}
\hline Merge (mins) & Logical operator & Edge default & Accuracy & Precision & Recall & F1 \\
\hline 5 & AND & Non-wear time & $1.0( \pm 0.0003)$ & $0.9995( \pm 0.0019)$ & $1.0( \pm 0.0)$ & $0.9997( \pm 0.0013)$ \\
\hline 4 & AND & Non-wear time & $0.9997( \pm 0.0013)$ & $0.9995( \pm 0.0019)$ & $0.9889( \pm 0.0085)$ & $0.9941( \pm 0.0062)$ \\
\hline 4 & OR & Wear time & $0.999( \pm 0.0026)$ & $0.9551( \pm 0.0168)$ & $0.9993( \pm 0.0022)$ & $0.9767( \pm 0.0123)$ \\
\hline 3 & OR & Wear time & $0.9989( \pm 0.0026)$ & $0.9552( \pm 0.0168)$ & $0.9979( \pm 0.0038)$ & $0.9761( \pm 0.0124)$ \\
\hline 5 & OR & Wear time & $0.9989( \pm 0.0027)$ & $0.9498( \pm 0.0177)$ & $1.0( \pm 0.0)$ & $0.9742( \pm 0.0129)$ \\
\hline 3 & AND & Non-wear time & $0.9974( \pm 0.0041)$ & $1.0( \pm 0.0)$ & $0.8785( \pm 0.0265)$ & $0.9353( \pm 0.02)$ \\
\hline 2 & OR & Wear time & $0.9971( \pm 0.0044)$ & $0.9437( \pm 0.0187)$ & $0.9203( \pm 0.022)$ & $0.9319( \pm 0.0205)$ \\
\hline 1 & OR & Wear time & $0.9962( \pm 0.005)$ & $0.9158( \pm 0.0225)$ & $0.9037( \pm 0.0239)$ & $0.9097( \pm 0.0233)$ \\
\hline 3 & OR & Non-wear time & $0.9955( \pm 0.0054)$ & $0.828( \pm 0.0306)$ & $0.9979( \pm 0.0038)$ & $0.905( \pm 0.0238)$ \\
\hline 2 & OR & Non-wear time & $0.9954( \pm 0.0055)$ & $0.8244( \pm 0.0309)$ & $0.9971( \pm 0.0044)$ & $0.9026( \pm 0.0241)$ \\
\hline 1 & OR & Non-wear time & $0.9949( \pm 0.0058)$ & $0.8184( \pm 0.0313)$ & $0.9805( \pm 0.0112)$ & $0.8922( \pm 0.0252)$ \\
\hline 4 & OR & Non-wear time & $0.9948( \pm 0.0059)$ & $0.8045( \pm 0.0322)$ & $0.9993( \pm 0.0022)$ & $0.8913( \pm 0.0253)$ \\
\hline 5 & OR & Non-wear time & $0.9944( \pm 0.0061)$ & $0.7924( \pm 0.0329)$ & $1.0( \pm 0.0)$ & $0.8841( \pm 0.026)$ \\
\hline 2 & AND & Non-wear time & $0.9954( \pm 0.0055)$ & $1.0( \pm 0.0)$ & $0.7862( \pm 0.0333)$ & $0.8803( \pm 0.0264)$ \\
\hline 1 & AND & Non-wear time & $0.994( \pm 0.0063)$ & $1.0( \pm 0.0)$ & $0.7202( \pm 0.0364)$ & $0.8373( \pm 0.03)$ \\
\hline 5 & AND & Wear time & $0.9908( \pm 0.0077)$ & $0.9991( \pm 0.0025)$ & $0.5736( \pm 0.0401)$ & $0.7288( \pm 0.0361)$ \\
\hline 4 & AND & Wear time & $0.9906( \pm 0.0078)$ & $0.9991( \pm 0.0025)$ & $0.5625( \pm 0.0403)$ & $0.7197( \pm 0.0365)$ \\
\hline 3 & AND & Wear time & $0.9888( \pm 0.0086)$ & $1.0( \pm 0.0)$ & $0.4763( \pm 0.0405)$ & $0.6452( \pm 0.0388)$ \\
\hline 2 & AND & Wear time & $0.9887( \pm 0.0086)$ & $1.0( \pm 0.0)$ & $0.4742( \pm 0.0405)$ & $0.6433( \pm 0.0389)$ \\
\hline 1 & AND & Wear time & $0.9873( \pm 0.0091)$ & $1.0( \pm 0.0)$ & $0.4082( \pm 0.0399)$ & $0.5797( \pm 0.0401)$ \\
\hline
\end{tabular}

Table 1. The classification of accuracy, precision, recall, and F1 performance metrics when applying the new algorithm on $50 \%$ of the available data $(n=291 / 583)$ while exploring 20 combinations of hyperparameter values; $95 \%$ confidence intervals are shown between parentheses. Merge (mins) $=$ the merging of neighbouring candidate non-wear episodes to handle artificial movement. Logical operator = AND if both start and stop segments or OR if only one side of a candidate non-wear episode needs to be classified as true non-wear time to subsequently classify the candidate non-wear episode as an episode of true non-wear time. Edge default $=$ the default classification of a candidate non-wear episode that has no start or end segment, such cases that occur right at the beginning or end of the acceleration data and default to wear or non-wear time.

are remaining steps that involve traversing the raw triaxial data and handling the following cases: (i) artificial movement by merging neighbouring candidate non-wear episodes; (ii) inspecting two logical operators AND and OR to determine if the start and stop segments combined (i.e. AND) or a single side (i.e. OR) results in a better detection of true non-wear time; and (iii) candidate non-wear episodes at the beginning or end of the acceleration signal that have no preceding start or following end segment. Table 1 presents the classification performance of detecting true non-wear time episodes from a random sample of $50 \%$ (i.e. training data) of the participants from the gold-standard dataset when utilising the CNN v2 architecture with a window size of $3 \mathrm{~s}$ and exploring 20 combinations of hyperparameter values.

The best F1 score $(0.9997 \pm 0.0013)$ on the training set was achieved by: (i) merging neighbouring candidate non-wear episodes that are a maximum of 5 mins apart from each other, (ii) using the logical operator AND (meaning both start and stop segments need to be classified as non-wear time to subsequently classify the candidate non-wear episode into true non-wear time), and (iii) default start and stop segments to non-wear time when they occur right at the start or end of the acceleration signal. Using these hyperparameter values on the remaining $50 \%$ of our gold-standard dataset (i.e. test data) achieved similar results: accuracy of $0.9999( \pm 0.0006)$, precision of $1.0( \pm 0.0)$, recall of $0.9962( \pm 0.005)$, and F1 performance of $0.9981( \pm 0.0035)$. In summary, the proposed algorithm is able to achieve near perfect performance on the training and test dataset when detecting non-wear episodes, both in terms of the ability to correctly classify an episode as true non-wear time (high precision), as well as the ability to detect all available non-wear time episodes present in the dataset (high recall).

Non-wear time algorithm steps. Based on the results presented in Table 1, and the steps outlined in the "Methods" section, the complete algorithm is presented below.

Detect candidate non-wear episodes. Perform a forward pass through the raw acceleration signal and calculate the SD for each 1-min interval of each axis. If the standard deviation is $\leq 0.004 \mathrm{~g}$ for all axes, record this 1 -min interval as a candidate non-wear interval. After all of the 1-min intervals have been processed, merge consecutive 1-mins intervals into candidate non-wear episodes and record their start and stop timestamps.

Merge bordering candidate non-wear episodes. Merge candidate non-wear episodes that are no more than 5 mins apart and record their new start and stop timestamps. This step is required to capture artificial movement that would typically break up two or more candidate non-wear episodes in close proximity. 


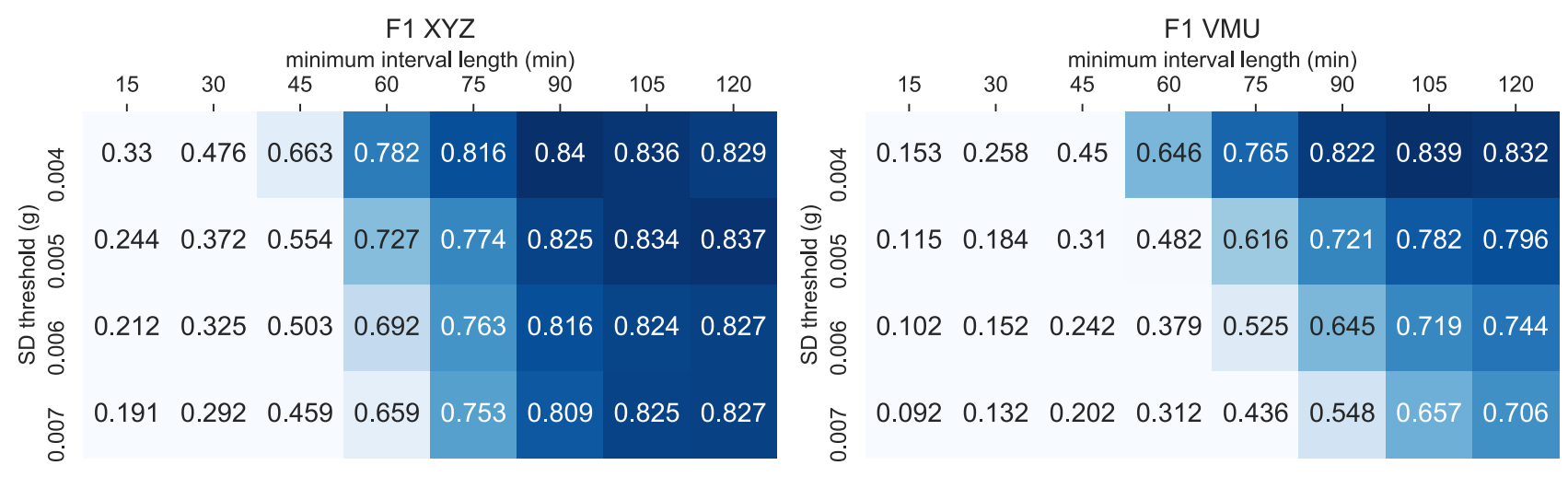

Figure 4. The F1 classification performance of the $X Y Z$ baseline algorithm (left), and the VMU baseline algorithm (right). Note that a SD threshold of $0.003 \mathrm{~g}$ performed poorly as it is below the accelerometer noise level and is therefore not shown. See Supplementary Figs. S4 and S5 for accuracy, precision, and recall scores.
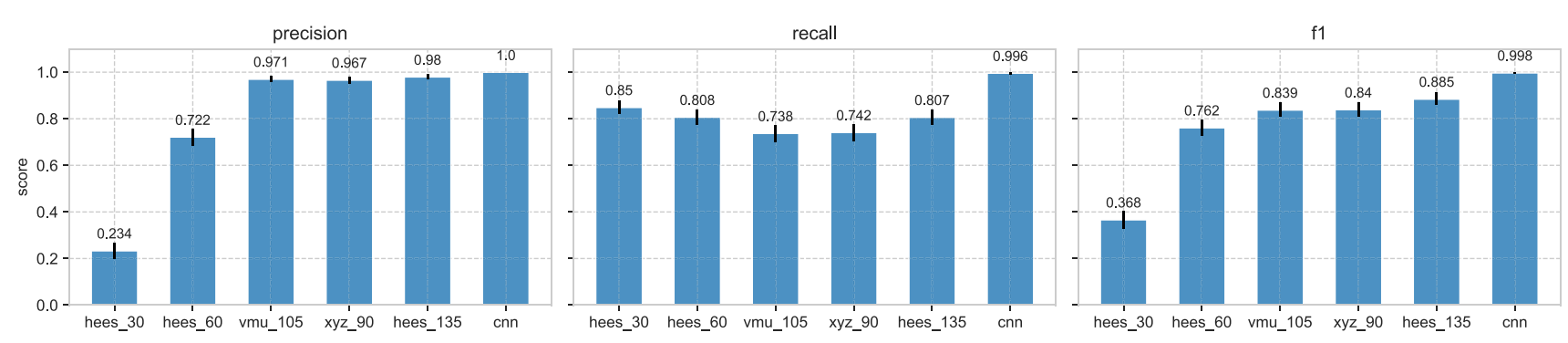

Figure 5. A comparison of the classification performance metrics of the best performing baseline models XYZ_90 (i.e. calculating the standard deviation of the three individual axes and an interval length of 90 mins), VMU_105 (i.e. calculating the standard deviation of the VMU and an interval length of 105 mins), the HEES_30 algorithm with a 30 mins interval, the HEES_60 with a 60 mins interval, the HEES_135 with tuned hyperparameters and a 135 mins interval, and the proposed CNN algorithm. Error bars represent the 95\% confidence interval.

Detect the edges of candidate non-wear episodes. Perform a backward pass with a 1-s step size through the acceleration data from the start timestamp of a candidate non-wear episode and calculate the SD for each axis. The same is applied to the stop timestamps with a forward pass and a step size of $1 \mathrm{~s}$. If the SD of all axes is $\leq 0.004$ $g$, include the 1-s interval in the candidate non-wear episode and record the new start or stop timestamp. Repeat until the SD of the 1-s interval does not satisfy the SD threshold $\leq 0.004 \mathrm{~g}$. This results in the resolution of the edges now being recorded on a 1-s resolution.

Classifying the start and stop windows. For each candidate non-wear episode, extract the start and stop segment with a window length of $3 \mathrm{~s}$ to create input features for the CNN classification model. For example, if a candidate non-wear episode has a start timestamp of $t_{\text {start }}$, a feature matrix is created as $\left(t_{\text {start-w }}: t_{\text {start }}\right)$ x 3 axes (where $w$ $=3 \mathrm{~s}$ ), resulting in an input feature with dimensions of $300 \times 3$ for $100 \mathrm{~Hz}$ data. If both start and stop features (i.e. logical AND) are classified (through the CNN model) as non-wear time, the candidate non-wear episode can be considered true non-wear time. If $t_{\text {start }}$ is $t=0$, or $t_{\text {end }}$ is at the end of the acceleration data, those candidate non-wear episodes do not have a preceding or following window to extract features from, the start or stop can be, by default, classified as non-wear time.

Evaluation against baseline and existing non-wear algorithms. Figure 4 presents the F1 performance of the two baseline algorithms as outlined in the "Methods" section. Figures S4 and S5 in the Supplementary Information provides, in additional to the F1 scores, the performance metrics accuracy, precision, and recall for the $X Y Z$ and $V M U$ baseline algorithms respectively. As shown in Fig. 4, increasing the SD threshold from $0.004 \mathrm{~g}$ to a higher value resulted in an F1 performance loss for both the $X Y Z$ and $V M U$ baseline algorithms. The XYZ algorithm achieved the highest F1 score with a SD threshold of $\leq 0.004 \mathrm{~g}$ and an interval length of 90 mins; further increasing the interval to 105 or $120 \mathrm{mins}$ is associated with lower F1 scores, 0.836 and 0.829 respectively. The use of longer intervals resulted in non-wear episodes that were shorter than the interval to not be detected, which caused the recall score to be lower. At the same time, shortening the interval length resulted in a higher recall but a lower precision score (Supplementary Fig. S4). The optimal F1 score for the VMU baseline algorithm (0.839) was achieved with a SD threshold of $\leq 0.004 \mathrm{~g}$ and an interval length of 105 mins. The VMU algorithm shows a similar pattern to the $X Y Z$ algorithm with respect to balancing the trade-off between capturing more non-wear time (higher recall) with shorter intervals, at a cost of lowering the precision (Supplementary Fig. S5), 
or using longer intervals to detect less overall non-wear time (lower recall), in favour of being more certain that the inferred non-wear time is true non-wear time (higher precision).

Besides baseline algorithms, the raw non-wear algorithms developed by van Hees and colleagues with a 30 mins interval ${ }^{19}$ (HEES_30), a later published 60-mins interval ${ }^{20}$ (HEES_60), and one with a 135-mins interval and tuned hyperparameters ${ }^{14}$ (HEES_135) were also evaluated. Figure 5 presents an overview of the obtained classification performance data (precision, recall, and F1) of all evaluated non-wear algorithms against the proposed CNN algorithm. The HEES_135 algorithm with tuned hyperparameters outperformed the default HEES_30 and HEES_60 algorithms with an F1 score of 0.885. In addition, HEES_135 outperformed the best performing baseline algorithm, $X Y Z(F 1=0.84)$ with a 90 -mins interval (i.e. XYZ_90), as well as the VMU baseline algorithm, $(\mathrm{F} 1=0.839)$ with a $105-\mathrm{min}$ interval (i.e. $\left.V M U_{-} 105\right)$. However, the proposed CNN method outperformed all evaluated non-wear algorithms with a near perfect F1 score of 0.998 .

\section{Discussion}

In this paper, we proposed a novel algorithm to detect non-wear time from raw accelerometer data through the use of deep convolutional neural networks and insights adopted from the field of physical activity type recognition ${ }^{22-29}$. We utilised a previously constructed gold-standard dataset ${ }^{14}$ with known episodes of true non-wear time from 583 participants, and were able to achieve an F1 score of 0.998 , outperforming baseline algorithms and existing non-wear algorithms ${ }^{19,20}$.

The main advantage of the proposed algorithm is the absence of a minimum interval (e.g. 30 mins or 60 mins) in which a specific metric (e.g. SD) needs to be below a threshold value (e.g. $4 \mathrm{mg}$ ). Currently, all existing raw and epoch-based non-wear algorithms adopt a minimum interval and have to balance between precision and recall $^{14}$. In other words, a short interval increases the detection of all present non-wear time (higher recall), at the cost of incorrectly inferring wear time as non-wear time (lower precision). Alternatively, a longer interval decreases the detection of all present non-wear time (lower recall), but those detections are more certain to be true non-wear time (higher precision); this trade-off has been discussed at length in our previously published study $^{14}$. A similar finding is shown in Fig. 5, where HEES_30 achieved a better recall score (0.85) compared to HEES_60 (0.808) but performed poorly on the precision metric (0.234) in comparison to HEES_60 (0.772); here both HEES_30 and HEES_60 are identical algorithms with the only difference being the interval length.

In line with the above, a larger interval caused a stronger increase in precision scores than a decrease in recall score, subsequently resulting in an overall higher F1 score. In other words, larger intervals perform better in the overall detection and correct classification of both wear and non-wear time; which is what is captured by the F1 metric. In fact, as can be seen from Fig. 5, some of the evaluated baseline and existing non-wear algorithms were able to achieve very high precision scores of 0.971 (VMU_105), 0.967 (XYZ_90), and 0.98 (HEES_135). These results show that the evaluated algorithms can be near perfect in their ability to correctly classify an episode into true non-wear time without too many false positives (type I error). However, a major drawback is that longer intervals cannot detect episodes of non-wear time shorter than the interval. This is a major shortcoming of nonwear algorithms to date and causes their ability to detect all the available non-wear time within the data to be sub-optimal. As a direct consequence, true non-wear episodes shorter than the interval will be inferred as wear time, which can result in an increase of false negatives or type II errors. For datasets with a high frequency of short non-wear time episodes, this can cause derived PA summary statistics to be incorrect, especially summary statistics that are relative to the amount of activity detected. Our proposed CNN algorithm did not perform better on the precision score, however, by not relying on an interval, it was able to detect even the shortest episodes of non-wear time; this enabled the recall score to be high and, as a consequence, resulted in a higher F1 score as well.

As per our analysis, the CNN v2 architecture achieved the highest F1 score on the training, validation, and test set, making it superior to the CNN v3 architecture with a higher number of convolutional kernels and filters in each layer. The CNN v3 architecture starts to overfit to the training data and results in lower classification performance on the validation set; which cause model training to stop with early stopping enabled. Although not explored, regularization methods such as several dropout layers ${ }^{38}$ will likely prevent overfitting and can potentially increase the performance of the $\mathrm{CNN} v 3$ architecture. The $\mathrm{CNN}$ v 4 architecture with max pooling layers, which essentially down-samples the features, shows sub-optimal performance compared to the architecture without max pooling layers (i.e., CNN v2). This effect is in line with previous research where max pooling layers weakens the classification performance of the model $^{34}$.

Hyperparameter values. The explored hyperparameter "Edge default" has the risk of being dataset specific, despite our efforts to train on $50 \%$ of the data and test on the remaining, unseen, $50 \%$. Its default classification to wear time for episodes without a start or stop segment (those at the beginning or end of the activity data) can be linked to the study protocol and might not translate to other datasets unquestionably. For example, if accelerometers are initialised and start recording before given to the participants, it might be assumed that the recordings after initialisation are non-wear time since the accelerometer still needs to be worn, either shortly thereafter or at a later stage when sent to participants via postal mail. In such cases, a preceding segment can default to non-wear time. However, if accelerometers are initialised to record at midnight (i.e. 00:00), and worn before recording starts, defaulting to non-wear time might not be automatically correct. For example, the participant might sleep before recording starts and, as a result, can obtain a recording at $t=0$ that does not exceed the $\leq 0.004 \mathrm{~g}$ SD threshold; in such cases, defaulting to non-wear time would be incorrect.

Additionally, the hyperparameter "merge (mins)", that merges nearby candidate non-wear episodes to capture and include artificial movement, could unintentionally merge true non-wear and wear time episodes if they occur very close to each other (i.e. $<5$ mins). For example, if the accelerometer was worn during sleep but removed right after waking up, two candidate non-wear episodes could be detected and merged incorrectly. Although this 
did not occur in our dataset, reducing the "merge (mins)" hyperparameter to 4 mins would further reduce the risk of incorrect merging and, given our results, still achieved an F1 score of $0.9941( \pm 0.0062)$ on our training set (Table 1).

Limitations and future research. Care must be given to the nature of the PA patterns that we detected in the preceding and following windows of a candidate non-wear episode. As per our results, the CNN model was able to differentiate true non-wear time from wear time segments with near perfect performance based on features taken $3 \mathrm{~s}$ before the episode started and $3 \mathrm{~s}$ after the episode ended. Longer feature segments, of 4 or 5 s, yielded similar statistical results since, effectively, a shorter segment remains a subset of a longer segment. The activity patterns of taking off the accelerometer (preceding feature) or putting it back on (following feature) were distinguished from activity patterns that proceeded or followed a candidate non-wear episode that was deemed wear time. In the latter, the CNN model learned patterns that were associated with movement during episodes of no activity, but those where the accelerometer was still worn. Such patterns are, for example, rotating the body during sleep, and during sedentary time, changing sitting positions. All learned patterns were captured from an accelerometer positioned on the right hip. Moreover, the accelerometer was mounted with an elastic waist belt, which could have an additional effect on the learned movement patterns, compared to having a (belt) clip or another way of mounting the accelerometer on the hip. We further suspect the activity patterns to be different for accelerometers positioned on the wrist, since this can be associated with higher movement variability ${ }^{4}$.

A natural next step would thus be to employ a similar approach of detecting non-wear time through activity type recognition by means of deep neural networks for accelerometers positioned at different locations, such as the wrist. With the use of raw accelerometer data, we are confident that our algorithm is invariant to different types of accelerometer brands positioned on the hip, even when data was sampled at different frequencies, as they can easily be resampled to $100 \mathrm{~Hz}^{39}$. However, other accelerometers may have a different standard deviation threshold value to detect episodes of no activity. Our dataset contained accelerometer data from the ActiGraph wGT3X-BT, which is the most commonly used accelerometer for PA studies ${ }^{8,17}$. A careful analysis revealed that a threshold of $0.004 \mathrm{~g}$ is close to the accelerometer's noise level and sufficiently low enough to detect episodes of no activity. This threshold value should, however, be explored for other accelerometers when using our proposed algorithm.

Although raw accelerometer data has shown promising results in terms of detecting non-wear time, care must be given to sources of uncertainty and error when handling and processing raw accelerometer data. As previously mentioned, resampling to other sample frequencies can be considered, for example 30 to $100 \mathrm{~Hz}$. However, the effects of resampling are ill understood. Sampling algorithms that interpolate make underlying assumptions with respect to interpolation errors and coefficient quantization errors, and as a result, are limited in their ability to correctly resample ${ }^{39}$. How resampling effects the typically multi-axial acceleration values of accelerometers is an interesting directive to explore. Another potential source of error is the acceleration sensor calibration. Typically, this calibration is done during manufacturing in which the recorded acceleration values should not exceed the local gravitational acceleration during non-movement episodes. However, in some cases it is worth re-calibrating the accelerometer data with a process called auto-calibration ${ }^{40}$. How auto-calibration effects the activity patterns which are required for accurate non-wear detection is worth exploring as well.

\section{Conclusion}

In this paper, we proposed a novel algorithm that utilises a deep convolutional neural network to detect the activity types of taking off the accelerometer and placing it back on to enable the detection of non-wear time from raw accelerometer data. Though current raw non-wear time algorithms show promising results in terms of precision scores, their employed interval prevents them from detecting non-wear time shorter than this interval, resulting in a sub-optimal recall score. By classifying activity types, our proposed algorithm does not employ a minimum interval and allows for non-wear time detection of any duration, even as short as a single minute. As per our results, this significantly increased the recall ability and led to a near perfect F1 score (0.998) on our gold-standard test dataset. Although our algorithm was developed for movement associated with a hip-worn accelerometer, future research can be directed at training a CNN for movement associated with a wrist-worn accelerometer, including the optimisation of our algorithm's hyperparameters.

\section{Data availability}

The legal restriction on data availability are set by the Tromsø Study Data and Publication Committee in order to control for data sharing, including publication of datasets with the potential of reverse identification of deidentified sensitive participant information. The data can however be made available from the Tromsø Study upon application to the Tromsø Study Data and Publication Committee. All Python code that supports this study is openly available on S.S.s GitHub page at https://github.com/shaheen-syed/ActiGraph-ActiWave-Analysis. A Python implementation of the CNN non-wear algorithm can be found at https://github.com/shaheen-syed/ CNN-Non-Wear-Time-Algorithm.

Received: 26 May 2020; Accepted: 5 April 2021

Published online: 23 April 2021

\section{References}

1. Doherty, A. et al. Large scale population assessment of physical activity using wrist worn accelerometers: The UK biobank study. PLoS ONE 12, e0169649. https://doi.org/10.1371/journal.pone.0169649 (2017). 
2. Dowd, K. P. et al. A systematic literature review of reviews on techniques for physical activity measurement in adults: A DEDIPAC study. Int. J. Behav. Nutr. Phys. Act. 15, 2019. https://doi.org/10.1186/s12966-017-0636-2 (2018).

3. Loyen, A. et al. Sedentary time and physical activity surveillance through accelerometer pooling in four European countries. Sports Med. 47, 1421-1435. https://doi.org/10.1007/s40279-016-0658-y (2017).

4. Montoye, A. H. et al. Raw and count data comparability of hip-worn actigraph GT3X+ and link accelerometers. Med. Sci. Sports Exerc. 50, 1103-1112. https://doi.org/10.1249/MSS.0000000000001534 (2018).

5. Troiano, R. P., McClain, J. J., Brychta, R. J. \& Chen, K. Y. Evolution of accelerometer methods for physical activity research. Br. J. Sports Med. 48, 1019-1023. https://doi.org/10.1136/bjsports-2014-093546 (2014).

6. Bassett, D. R., Rowlands, A. \& Trost, S. G. Calibration and validation of wearable monitors. Med. Sci. Sports Exerc. 44, S32-S38. https://doi.org/10.1249/MSS.0b013e3182399cf7 (2012).

7. Choi, L., Ward, S. C., Schnelle, J. F. \& Buchowski, M. S. Assessment of wear/nonwear time classification algorithms for triaxial accelerometer. Med. Sci. Sports Exerc. 44, 2009-2016, https://doi.org/10.1249/MSS.0b013e318258cb36 (2012).

8. Migueles, J. H. et al. Comparability of accelerometer signal aggregation metrics across placements and dominant wrist cut points for the assessment of physical activity in adults. Sci. Rep. 9, 18235. https://doi.org/10.1038/s41598-019-54267-y (2019).

9. Lee, I.-M. \& Shiroma, E. J. Using accelerometers to measure physical activity in large-scale epidemiological studies: Issues and challenges. Br. J. Sports Med. 48, 197-201. https://doi.org/10.1136/bjsports-2013-093154 (2014).

10. Migueles, J. H. et al. Accelerometer data collection and processing criteria to assess physical activity and other outcomes: A systematic review and practical considerations. Sports Med. 47, 1821-1845. https://doi.org/10.1007/s40279-017-0716-0 (2017).

11. Choi, L., Liu, Z., Matthews, C. \& Buchowski, M. Validation of accelerometer wear and nonwear time classification algorithm. Med. Sci. Sports Exerc. 43, 357-364. https://doi.org/10.1249/MSS.0b013e3181ed61a3 (2011).

12. Hecht, A., Ma, S., Porszasz, J. \& Casaburi, R. Methodology for using long-term accelerometry monitoring to describe daily activity patterns in COPD. COPD 6, 121-129, https://doi.org/10.1080/15412550902755044 (2009).

13. Troiano, R. P. et al. Physical activity in the United States measured by accelerometer. Med. Sci. Sports Exerc. 40, 181-188. https:// doi.org/10.1249/mss.0b013e31815a51b3 (2007).

14. Syed, S., Morseth, B., Hopstock, L. A. \& Horsch, A. Evaluating the performance of raw and epoch non-wear algorithms using multiple accelerometers and electrocardiogram recordings. Sci. Rep. 10, 5866. https://doi.org/10.1038/s41598-020-62821-2 (2020).

15. Aadland, E., Andersen, L. B., Anderssen, S. A. \& Resaland, G. K. A comparison of 10 accelerometer non-wear time criteria and logbooks in children. BMC Public Health 18, 323. https://doi.org/10.1186/s12889-018-5212-4 (2018).

16. Jaeschke, L. et al. $24 \mathrm{~h}$-accelerometry in epidemiological studies: Automated detection of non-wear time in comparison to diary information. Sci. Rep. 7, 2227. https://doi.org/10.1038/s41598-017-01092-w (2017).

17. Knaier, R., Höchsmann, C., Infanger, D., Hinrichs, T. \& Schmidt-Trucksäss, A. Validation of automatic wear-time detection algorithms in a free-living setting of wrist-worn and hip-worn ActiGraph GT3X+. BMC Public Health 19, 244. https://doi.org/10. $1186 / \mathrm{s} 12889-019-6568-9$ (2019).

18. Vanhelst, J. et al. Comparison and validation of accelerometer wear time and non-wear time algorithms for assessing physical activity levels in children and adolescents. BMC Med. Res. Methodol. 19, 72. https://doi.org/10.1186/s12874-019-0712-1 (2019).

19. van Hees, V. T. et al. Estimation of daily energy expenditure in pregnant and non-pregnant women using a wrist-worn tri-axial accelerometer. PLoS ONE 6, e22922. https://doi.org/10.1371/journal.pone.0022922 (2011).

20. van Hees, V. T. et al. Separating movement and gravity components in an acceleration signal and implications for the assessment of human daily physical activity. PLoS ONE 8, e61691. https://doi.org/10.1371/journal.pone.0061691 (2013).

21. Ahmadi, M. N., Nathan, N., Sutherland, R., Wolfenden, L. \& Trost, S. G. Non-wear or sleep? Evaluation of five non-wear detection algorithms for raw accelerometer data. J. Sports Sci. 38, 399-404. https://doi.org/10.1080/02640414.2019.1703301 (2020).

22. Bayat, A., Pomplun, M. \& Tran, D. A. A study on human activity recognition using accelerometer data from smartphones. Procedia Comput. Sci. 34, 450-457. https://doi.org/10.1016/j.procs.2014.07.009 (2014).

23. Chatzaki, C., Pediaditis, M., Vavoulas, G. \& Tsiknakis, M. Human daily activity and fall recognition using a smartphone's acceleration sensor. In Röcker, C., O’Donoghue, J., Ziefle, M., Helfert, M. \& Molloy, W. (eds.) Communications in Computer and Information Science, vol. 736 of Communications in Computer and Information Science, 100-118, https://doi.org/10.1007/978-3-319-62704-5_7 (Springer International Publishing, 2017).

24. Kwapisz, J. R., Weiss, G. M. \& Moore, S. A. Activity recognition using cell phone accelerometers. ACM SIGKDD Explor. Newsl. 12, 74. https://doi.org/10.1145/1964897.1964918 (2011).

25. Skotte, J., Korshøj, M., Kristiansen, J., Hanisch, C. \& Holtermann, A. Detection of physical activity types using triaxial accelerometers. J. Phys. Act. Health 11, 76-84. https://doi.org/10.1123/jpah.2011-0347 (2014).

26. Willetts, M., Hollowell, S., Aslett, L., Holmes, C. \& Doherty, A. Statistical machine learning of sleep and physical activity phenotypes from sensor data in 96,220 UK Biobank participants. Sci. Rep. 8, 7961. https://doi.org/10.1038/s41598-018-26174-1 (2018).

27. Zeng, M. et al. Convolutional neural networks for human activity recognition using mobile sensors. In Proceedings of the 6th International Conference on Mobile Computing, Applications and Services, vol. 6, 197-205, https://doi.org/10.4108/icst.mobicase. 2014.257786 (ICST, 2014).

28. Santos, G. et al. Accelerometer-based human fall detection using convolutional neural networks. Sensors 19, 1644. https://doi.org/ 10.3390/s19071644 (2019).

29. Shoaib, M., Bosch, S., Incel, O., Scholten, H. \& Havinga, P. Complex human activity recognition using smartphone and wrist-worn motion sensors. Sensors 16, 426. https://doi.org/10.3390/s16040426 (2016).

30. Jacobsen, B. K., Eggen, A. E., Mathiesen, E. B., Wilsgaard, T. \& Njolstad, I. Cohort profile: The Tromso study. Int. J. Epidemiol. 41, 961-967. https://doi.org/10.1093/ije/dyr049 (2012).

31. Sagelv, E. H. et al. Physical activity levels in adults and elderly from triaxial and uniaxial accelerometry. The Tromsø study. PLoS ONE 14, e0225670. https://doi.org/10.1371/journal.pone.0225670 (2019).

32. LeCun, Y., Bengio, Y. \& Hinton, G. Deep learning. Nature 521, 436-444. https://doi.org/10.1038/nature14539 (2015)

33. Lee, S., Yoon, S.M. \& Cho, H. Human activity recognition from accelerometer data using Convolutional Neural Network. In 2017 IEEE International Conference on Big Data and Smart Computing (BigComp), 131-134, https://doi.org/10.1109/BIGCOMP.2017. 7881728 (IEEE, 2017).

34. Ordóñez, F. \& Roggen, D. Deep convolutional and LSTM recurrent neural networks for multimodal wearable activity recognition. Sensors 16, 115. https://doi.org/10.3390/s16010115 (2016).

35. Kingma, D. P. \& Ba, J. Adam: A method for stochastic optimization. In 3rd International Conference on Learning Representations, ICLR 2015 - Conference Track Proceedings, 1-15 (2014). arXiv:1412.6980.

36. Abadi, M. et al. TensorFlow: A system for large-scale machine learning. In 12th USENIX Symposium on Operating Systems Design and Implementation (OSDI 16), 265-283 (2016).

37. van Hees, V. T. et al. GGIR: Raw Accelerometer data analysis, https://doi.org/10.5281/zenodo.1051064 (2019).

38. Srivastava, N., Hinton, G., Krizhevsky, A., Sutskever, I. \& Salakhutdinov, R. Dropout: A simple way to prevent neural networks from overfitting. J. Mach. Learn. Res. 15, 1929-1958 (2014).

39. Smith, J. O. Digital Audio Resampling Home Page (2002).

40. van Hees, V. T. et al. Autocalibration of accelerometer data for free-living physical activity assessment using local gravity and temperature: an evaluation on four continents. JJ. Appl. Physiol. 117, 738-744. https://doi.org/10.1152/japplphysiol.00421.2014 (2014). 


\section{Acknowledgements}

This work was supported by the High North Population Studies, UiT The Arctic University of Norway. The publication charges for this article have been funded by a grant from the publication fund of UiT The Arctic University of Norway.

\section{Author contributions}

Study concept and design: S.S. Data collection: B.M., L.A.H. Analysis and interpretation of data: S.S. Drafting of the manuscript: S.S. Critical revision of the manuscript: All authors.

\section{Competing interests}

The authors declare no competing interests.

\section{Additional information}

Supplementary Information The online version contains supplementary material available at https://doi.org/ 10.1038/s41598-021-87757-z.

Correspondence and requests for materials should be addressed to S.S.

Reprints and permissions information is available at www.nature.com/reprints.

Publisher's note Springer Nature remains neutral with regard to jurisdictional claims in published maps and institutional affiliations.

(c) (i) Open Access This article is licensed under a Creative Commons Attribution 4.0 International License, which permits use, sharing, adaptation, distribution and reproduction in any medium or format, as long as you give appropriate credit to the original author(s) and the source, provide a link to the Creative Commons licence, and indicate if changes were made. The images or other third party material in this article are included in the article's Creative Commons licence, unless indicated otherwise in a credit line to the material. If material is not included in the article's Creative Commons licence and your intended use is not permitted by statutory regulation or exceeds the permitted use, you will need to obtain permission directly from the copyright holder. To view a copy of this licence, visit http://creativecommons.org/licenses/by/4.0/.

(C) The Author(s) 2021 